\title{
Lean Stability Approaches in Pharmaceutical Product Development, Registration, and Post-marketing CMC Change Management; Industry Survey Insights
}

\author{
Donnie Pulliam ${ }^{1}$ (D) Megan McMahon ${ }^{2} \cdot$ Brian P. Regler $^{3} \cdot$ Susan Smith ${ }^{4} \cdot$ Cheryl Martin $^{5} \cdot$ Yelizaveta Babayan $^{6}$. \\ Cherokee Hoaglund-Hyzer ${ }^{6} \cdot$ Michael Lesslie $^{7} \cdot$ Fenghe Qiu $^{8} \cdot$ Eric Adamec $^{6} \cdot$ Jin Wang $^{9} \cdot$ Pat Forenzo $^{10}$. \\ Chaitanya S. Wannere ${ }^{11}$. Ping Zhuang ${ }^{11}$. Lars Gruenig ${ }^{12}$
}

Accepted: 31 January 2021 / Published online: 23 June 2021

(c) The Author(s) 2021

\begin{abstract}
Purpose This paper summarizes results from a recent pharmaceutical industry survey to understand the adoption status of science- and risk-based approaches in pharmaceutical stability (lean stability) in regulatory submissions across the full pharmaceutical product life cycle and to ascertain whether companies are deriving their stability strategies based on scientific risk or applying generic strategies.

Methods A comprehensive survey was distributed to pharmaceutical companies affiliated with the International Consortium for Innovation and Quality in Pharmaceutical Development (IQ Consortium) to solicit non-proprietary responses pertaining to regulatory strategies for stability monitoring across the product life cycle, perceived risks, and health authority feedback. Results Among the 19 responding companies, utilization of lean stability approaches varied with respect to (1) phase of product life cycle, (2) specific product quality attributes cited in effective ICH guidances or which demonstrated shelf-life limiting stability trends, and (3) the degree of perceived risk of rejection by health authorities based upon companies' degree of experience from previous regulatory submissions.

Conclusion Continued collaboration between the pharmaceutical industry and health authorities - through case studies and revision/harmonization of regulatory guidances - will be essential for continued implementation of lean stability strategies to accelerate delivery of innovative medicines to the clinic and to the market.
\end{abstract}

Keywords Lean stability $\cdot$ Risk-based stability $\cdot$ Pharmaceutical development $\cdot$ Regulatory affairs $\cdot$ Industry survey $\cdot$ Pharmaceuticals $\cdot$ Risk assessment

Donnie Pulliam

donnie.pulliam@biogen.com

1 Biogen Inc., 5000 Davis Drive, NC 27709 Durham, USA

2 Pfizer Inc., Eastern Point Road, CT 06340 Groton, USA

3 Ferring Pharmaceuticals, 100 Interpace Pkwy, Parsippany, NJ 07054, USA

4 AstraZeneca, Silk Road Business Park, Macclesfield, Cheshire SK10 2NA, UK

5 Baxter Healthcare Corporation, 25212 W. Illinois Route 120, Round Lake, IL 60073, USA

6 Eli Lilly \& Company, Lilly Corporate Center, Indianapolis, IN 46285, USA
7 AbbVie Inc., 1 N. Waukegan Road, IL 60064 North Chicago, USA

8 Boehringer Ingelheim, 900 Ridgebury Road, Ridgefield, CT 06877, USA

9 Genentech, 410 Allerton Ave, South San Francisco, CA 94080, USA

10 Novartis, One Health Plaza, East Hanover, NJ 07936-1080, USA

11 Merck \& Co., Inc., 126 E. Lincoln Avenue, NJ 07065 Rahway, USA

12 Novartis, Via Balestra, 6600 Locarno, Switzerland 


\section{Introduction}

Pharmaceutical manufacturers must assure regulatory agencies that therapeutic products distributed to patients conform to approved specifications throughout the expiration dating labeled on the product container. That assurance is founded upon an increasing understanding of manufacturing process capability and of product sensitivity to various chemical and physical degradation pathways evaluated during product development via stability monitoring of representative materials manufactured for clinical trials and prospective commercial distribution. The founding regulatory agencies of the International Conference on Harmonization (ICH: FDA, EMA, and PMDA) established clear expectations for product registration stability study design. ICH affiliation has grown since its inception in 1990 to 16 Members and 32 Observer health authorities [1]. These expectations (ICH Q1A [2] and Q5C [3]), together with other interdependent guidelines such as Q1D [4], Q1E [5], Q6A [6], and Q6B [7], provide confidence in data analyses which justify commercial product shelf-life. While most global health authorities accept strategies based on ICH guidelines, some have supplementary regulations which effectively raise hurdles to delivering innovative therapies globally. Industry-agency consortia have raised awareness to this problem; consequently, a growing number of health authorities are pursuing alignment.

Although ICH guidelines Q1A and Q5C specify the stability data package required to register a new product, filing stability protocols that vary relative to the recommended registration protocol design are allowed. Provided there is suitable scientific justification (including prior knowledge and sufficient supportive data), these guidelines permit the usage of reduced testing protocols for registration and commercial commitment (confirmatory and annual batch) long-term studies. Pharmaceutical companies may therefore propose reduced stability testing protocols justified by appropriate technical considerations and/or risk-assessment. These "lean stability" approaches can enable reallocation of company resources to accelerate the development of new therapies while continuing to ensure an appropriate level of quality monitoring for batches representing distributed clinical and commercial supplies.

Unfortunately, the pharmaceutical industry has encountered challenges to adopting lean stability approaches throughout product development and lifecycle, both from internal stakeholders and regulatory reviewers $[8,9]$. Due to the current divergence among global health authorities in relation to stability data requirements, companies may not be able to achieve lean stability approaches for products with the greatest potential to treat diseases. Industry has also observed varying positions from different reviewers within the same health authority.

In response to these circumstances, an IQ Consortium working group was formed with the purposes of gaining more understanding of (1) industry awareness to lean stability opportunities and approaches, (2) industry constraints to adopting lean stability approaches in product lifecycle management and regulatory submissions, and (3) agency receptivity to lean stability approaches included in clinical trial and marketing authorization applications. Since its beginning in 2016, the working group membership has consisted of a cross-functional team of 28 professionals in various CMC-related and regulatory affairs functions, representing 14 different IQ Consortium member pharmaceutical companies.

A survey was developed by the working group and was deployed to pharmaceutical companies that are members of the International Consortium for Innovation and Quality in Pharmaceutical Development (IQ Consortium) to understand the adoption status of lean stability approaches across the full pharmaceutical product life cycle. This article describes the development of the survey, summarizes key results from its distribution and discusses important insights from the survey results. Pharmaceutical industry readers of this article will be informed of the current state of adoption of lean stability approaches relative to the current global regulatory landscape. They will also be informed regarding actions they can take to advocate for greater usage of lean stability approaches and for more guidance from agencies regarding criteria for their implementation. Health authority readers of the article will be informed regarding efforts by pharmaceutical companies to apply science- and risk-based approaches to justify reduced stability monitoring that do not compromise quality or safety. They will also be informed of the current state of receptivity to lean stability strategies by other global health authorities.

\section{Definitions}

Accelerated Stability Modeling (ASM) and Accelerated Stability Assessment Program (ASAP) are specific approaches for performing risk-based predictive stability assessments. Science- and risk-based predictive stability is defined as short-term stability studies using high temperatures (and varying humidities) and mathematical modeling in order to predict stability at lower temperatures (and defined humidities) used for long-term storage conditions. These approaches lead to meaningful predictions of critical quality attributes for a DP or DS, packaged in a desired configuration, throughout its proposed shelf-life. 
Annual Stability Commitment Protocols are postapproval protocols registered as commitments to provide ongoing manufacturing consistency monitoring on other batch(es) than those referenced in commercial license applications.

$$
\mathrm{DP}=\text { Drug Product }
$$

DS $=$ Drug Substance or Active Pharmaceutical Ingredient (API)

Lean Stability Strategy(LSS) is a science- and riskbased stability approach focused on meaningful attributes and timepoints [10]. A lean stability strategy could include technical adjustments to stability protocols to improve efficiency and expedite results without impacting patient safety, efficacy, or quality of the product. Lean stability strategies could result in less frequent and/or delayed pull points, fewer stability conditions, and streamlined analytical test profiles that focus on the individual product's stability-related quality attributes (SRQAs) and ideally include only shelf-life limiting attributes (SLLAs). Lean strategies can facilitate the development and approval of new and improved medicines by emphasizing the key elements that contribute to quality, safety, and efficacy while deemphasizing elements that do not.

Modality is defined as a type of therapeutic drug distinguished by the manufacturing process for its active pharmaceutical ingredient. In this context, small molecule products produced via chemical synthesis processes are a distinct modality from biological/biotechnological products in which the active components are typically proteins and/or polypeptides produced from recombinant DNA-derived cell culture and purification.

Placebo to Match, or PTM, is defined as a dosage form (e.g., tablet, capsule, or any other drug form) that lacks any API and is manufactured in an image (including dimensions, color, and other attributes such as weight, shape, engraving, density, etc.) that matches active supplies of either a comparator product or an in-house developed product. The PTM may or may not be composed of the same inactive components as the active product or comparator.

Primary Stability Protocols are stability protocols used to collect stability data from representative commercial process batch(es) to be reported in commercial licensure applications.

Registration Stability Commitment Protocols are stability protocols registered in license applications committing to complete stability data collection on commercial process batch(es) referenced in commercial license applications, for purpose(s) such as confirming conformance to specifications through the desired/approved expiry period.
Risk-based Predictive Stability (examples include ASAP and ASM) is defined as accelerated stability studies using short-term high temperatures (and varying humidities) and modeling in order to predict stability at temperatures (and defined humidities) intended to be used for long-term storage conditions.

SLLAs or Shelf-life Limiting Attributesare attributes which will first breach their specified limit when stored at label conditions. They are a subset of stability-related quality attributes [11].

SRQAs or Stability-related Quality Attributes typically are a subset of quality attributes that either (1) directly or indirectly impact stability but may or may not impact other considerations (such as manufacture of the drug product) or (2) are attributes whose values may change on stability [11].

Stability Risk Assessment is a documented assessment of quality attributes that have the potential to impact retest date or shelf-life that is conducted during development and re-assessed as changes are introduced.

\section{Methods}

The survey consisted of an introductory prologue describing definitions of key terms and the survey's purpose and a total of 31 questions with a variety of customized subcomponents. These included:

- General information: qualitative description of the product types, modalities, and phases of development to enable interpretation of the responses to subsequent sections

- Stability risk assessments and protocol development

- Clinical development and submissions

- Registration (including primary stability studies/data and commitments)

- Post-approval changes

- Appendix, including questions related to stability monitoring practices for bulk materials, placebos, and collection and evaluation of in-use stability data

The survey was distributed in August 2017 to representatives of all pharmaceutical companies that were IQ Consortium members at that time. An intermediary was used for survey results distribution, which anonymized the identities of the contributing companies. Both qualitative and quantitative responses were compiled into a single database, and data transfer verification was conducted before assessing and interpreting the responses. Key data were transformed into graphics for presentation and publication, and working group discussions facilitated article co-authoring. 
Table 1 Pharmaceutical companies that responded to the IQ Consortium lean stability survey

\begin{tabular}{llll}
\hline Abbvie & Bayer & Genentech/Roche & Merck KGaA \\
Alkermes & Biogen & Gilead & Novartis \\
Amgen & Boehringer Ingelheim & GlaxoSmithKline & Pfizer \\
Astellas & Daiichi Sankyo & Ironwood & Teva \\
Baxter & Eli Lilly \& Company & MSD & \\
\hline
\end{tabular}

\section{Results}

\section{Respondent and Product Information}

Survey responses were received from a total of 19 pharmaceutical companies. Of these companies, four supplied two distinct survey responses: one pertaining to small molecule modality products and another relevant to biological/biotechnological products. Therefore, a total of 23 survey responses were received. Survey respondents indicated involvement in all phases of product development, commercialization, and postapproval life cycle management with a variety of pharmaceutical product forms. Survey responses were not specific to the product dosage form, unless indicated by a particular question. The list of respondents is provided in Table 1. Table 2 summarizes the variety of product presentations for which respondents apply specific stability strategies.

Nine respondents indicated involvement in large molecule (biologics products), and 17 respondents indicated involvement in small molecule development. Unless otherwise noted, results are reported in relation to the full number of 23 responses since, in most cases, the rationale for responses could not easily be decoupled with respect to modality due to the relatively low number of distinctmodality responses.

\section{Stability Risk Assessments and Protocol Development}

Based upon the survey responses, there appears to be a good acceptance of stability risk assessments (SRAs) among IQ member companies as 16 of the 19 respondents (84\%) indicated that SRAs are performed in their companies and an additional 2 respondents were planning on implementing SRAs in the near future. As illustrated in Fig. 1, over 50\% of these respondents communicate SRAs to the health agencies in their filings, mainly in late development. There are several ways to leverage SRAs in development, with the vast majority of companies using them to assess existing stability studies or to assign shelf-life attributes to products.

Responses from the survey indicate that stability protocols are highly customized, although templates do exist as a starting point. SRAs can be leveraged to tailor the stability program design, with equal applicability across all phases of development. Additionally, science and risk-based predictive stability approaches [12-14] such as ASAP or ASM, are used by a majority of the respondents in early development to drive the selection of temperature, relative humidity (\% RH), and/or packaging configurations in the design of stability protocols. Throughout product development and lifecycle, stability protocols are modified, if needed, due to changes in the DP formulation or manufacturing process, changes in DS process or form, packaging configurations, or the identification of stability trends.

Although the tests run on stability varied amongst the respondents, certain tests were always performed for both drug substance and drug product: description, impurities/ degradation products, and assay. Tests that were not always run but were typically performed included: water activity, physical characterization (for drug substances) and dissolution/disintegration (for drug products). While
Table 2 Survey respondent application of specific stability strategies to various pharmaceutical product forms

\begin{tabular}{lll}
\hline Pharmaceutical product form & $\begin{array}{l}\text { Respondents involved in develop- } \\
\text { ment and/or marketing }\end{array}$ & $\begin{array}{l}\text { Stability strategies } \\
\text { applied specific to prod- } \\
\text { uct form }^{\mathrm{a}}\end{array}$ \\
\hline Bulk DS & 19 & 14 \\
Solid oral dosage products & 19 & 15 \\
Solid parenteral dosage products & 17 & 8 \\
Liquid parenteral dosage products & 17 & 11 \\
Liquid oral dosage products & 14 & 8 \\
Inhaled products & 12 & 5 \\
Topical products & 10 & 5 \\
Aerosols & 4 & 3 \\
\hline
\end{tabular}

${ }^{\text {a }}$ Reflects the types of pharmaceutical product forms pertinent to stability strategies included in survey responses. This indicates differentiation between development and stability strategies among respondents 
Fig. 1 Applications of stability risk assessments

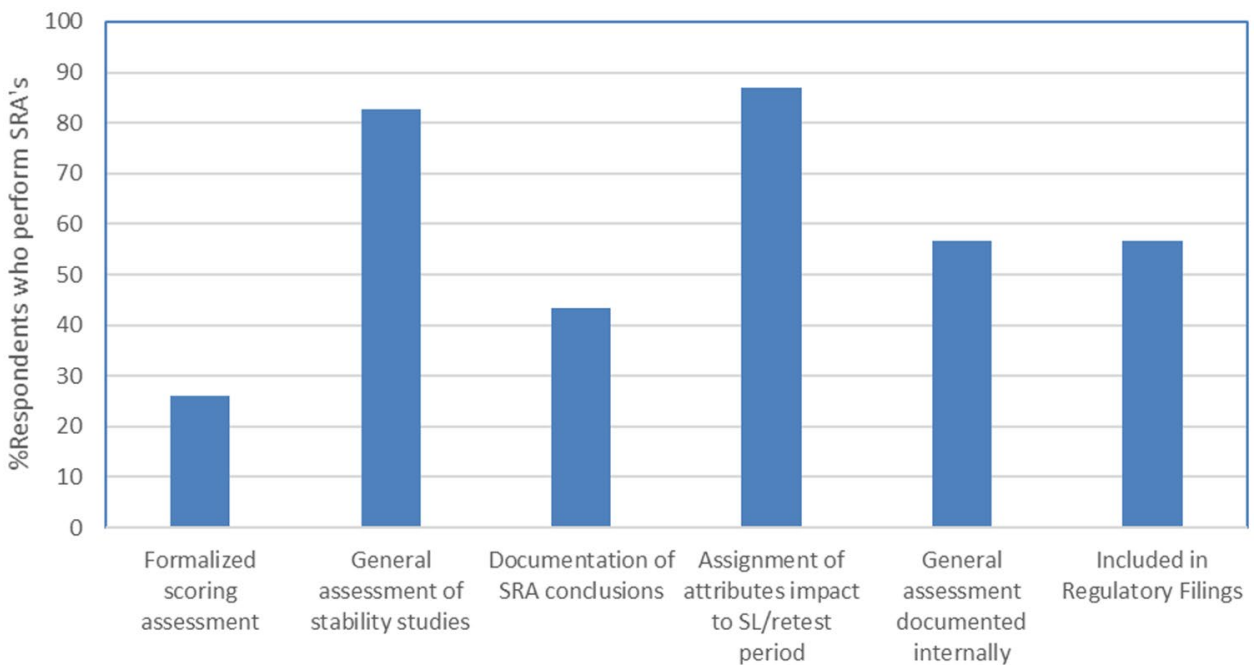

the overall goal for a stability program is to execute tests only for shelf-life limiting quality attributes, other tests were performed for a variety of reasons. These tests were usually performed in anticipation of regulatory queries, demonstration of comparability due to a DP/DS change or due to regulatory commitment(s).

\section{Clinical Phase Development and Submissions}

Several publications have described opportunities (case studies) for application of LSS during the clinical development lifecycle [8,15-17]. Inspired by these success stories and based on greater product understanding during earlier stages of development and availability of predictive tools, pharmaceutical companies have increased the use of lean strategies in their regulatory submissions.

Based on the survey conducted, $89 \%$ (17/19) of the companies surveyed have successfully utilized LSS in support of clinical trial application (CTA) submissions. Results from the survey indicate that LSS have been primarily used to support stability studies for drug product $(74 \%)$ and drug substance $(58 \%)$, with significant use for placebo (47\%), and relatively fewer examples for comparators $(32 \%)$. LSS have been successfully implemented to determine appropriate packaging configurations and storage conditions and to evaluate excipient compatibility/variability as well as changes in process, formulation, and/or packaging. Most frequently implemented strategies (16/19 companies) were those supported by regulatory guidance such as bracketing and matrixing strategies as outlined in ICH Q1D "Bracketing and Matrixing Designed for Stability Testing of New Drug Substances and Products."

In addition, stability data from development or clinical batches using materials that are representative (similar) to the manufacturing process of the clinical material have been used by the majority ( $>75 \%$ ) of companies in support of clinical material dating. In contrast, only $63 \%$ of the respondents indicated the use of accelerated and risk-based predictive stability (RBPS) approaches, which although have been gaining broader acceptance, are not directly acknowledged by regulatory guidance.

\section{Registration Protocols, Post-approval Commitments, and Post-approval Changes}

This section surveyed industry adoption of various scienceand risk-based approaches to stability testing in the different types of registration and commercial/lifecycle management phase stability protocols: primary stability protocols, registration stability commitment protocols, annual stability commitment protocols, and stability protocols to support post-approval changes. Protocols to support post-approval stability commitments and changes are executed to confirm the stability profile (retest/shelf-life) established by the primary stability protocol.

More than half of the 23 total responses acknowledged use of lean stability approaches in registration stability protocols already codified by or referenced in established ICH guidances such as bracketing $(n=17)$ and matrixing $(n=13)$ referred to in ICH Q1D. Evaluation of stability data collected to justify anticipated excursions from intended storage or distribution conditions was also cited often $(n=15)$ as a basis for justifying the inclusion of lean stability approaches in registration stability protocols. This accelerated and/or stressed condition stability data can demonstrate that product quality attribute(s) are unaffected by these conditions, which could provide justification for reduced testing frequency. Monitoring certain attributes only at annual time points, or only at initial and expiry 
period intervals, is used less frequently ( $n=11$ and $n=12$, respectively). Risk-based predictive stability (RBPS) approaches, which include accelerated stability assessment program (ASAP) Arrhenius calculation methods, were only cited in 8 of the responses.

Most respondents also reported including data from the following studies typically included in product registration dossiers: photostability $(n=21)$, thermal cycling or freeze/ thaw studies $(n=21)$, in-use stability evaluation $(n=21)$, and studies or interpretation of data simulating product exposure to high and low temperature excursions $(n=18)$. Responses also showed high occurrence of inclusion of data from vibrational/shipping studies $(n=15)$ and open dish/package breach studies $(n=12)$ in marketing applications for initial commercial product approval. Less frequently cited studies were admixture/compatibility testing $(n=6)$ and oxygen free/ reduced environment $(n=4)$. Only one company response indicated inclusion of data from oxidative environment study(ies) and another company indicated "stability after reconstitution, stability after dilution, patient convenience studies, and forced degradation (only for Brazil)."

Only ten of the 19 company respondents confirmed including lean stability approach(es) in the design of commercial commitment protocols to confirm conformance to shelf-life specifications through the expiry period. Supportive development data was the common factor cited in most $(n=11)$ responses as justification for using lean approaches, followed by data utilizing bracketing $(n=8$ responses), accelerated and/or RBPS data ( $n=8$ responses), data utilizing matrixing ( $n=7$ responses), and stability excursion data ( $n=5$ responses).

Figure 2 displays the list of product attributes proposed to be excluded from the filing since they are not shelf-life limiting attributes (SLLA(s)). The survey design did not accommodate for differentiating attributes for exclusion based on the distinct pharmaceutical forms listed in Table 2.

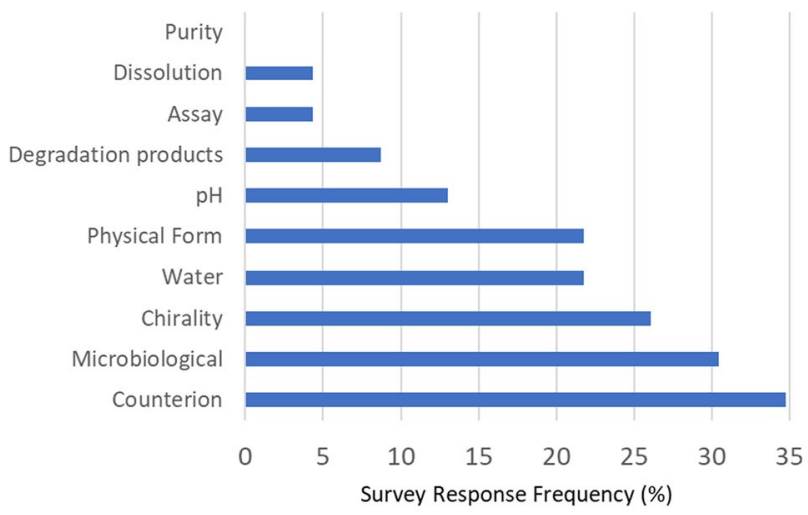

Fig. 2 Attributes proposed in product registration filings to be excluded from stability monitoring on the basis of not being shelf-life limiting attributes (SLLAs)
However, the relatively lower frequency of excluding attributes such as purity, dissolution, assay, and degradation products is not unexpected considering ICH Q1A and Q5C guidance documents specify their inclusion in stability monitoring of registration products and because these attributes often contribute to defining the product shelf-life.

Of the four companies supplying distinct responses for small molecule and large molecule modality products, three confirmed using lean stability approach(es) for both modalities. The other company respondent used lean stability approach(es) in biologic product application(s) but not in small molecule application(s). The specificity of these responses showed that attributes unique to small molecules and/or solid dosage forms, such as counterion and chirality, were non-applicable attribute choices for the large molecule-focused responses. While the number of modality-specific responses was relatively small, the large molecule responses showed relatively fewer attribute exclusion proposals in commercial commitment protocols to confirm expiry. Among the attributes shown in Fig. 2, only $\mathrm{pH}$ was cited in more than one large molecule modality response for exclusion from monitoring in registration batch stability through expiry.

Approximately $87 \%$ of those who responded indicated that science and risk-based approaches should be applied to annual stability batch protocols. Most respondents agreed that this could incorporate a reduction in test conditions, product presentation, frequency of testing, and attributes tested. However, application of lean stability concepts to post-approval commitment study designs seems to be practiced less widely. Survey responses indicate that this is due in part to industry mindsets based on perceived regulatory risks, and fear or uncertainty rooted in the lack of experience and expertise in lean stability and RBPS.

Results indicate that industry continues to use ICH Q1A and Q5C based approaches to support a post-approval change and is less willing to pursue a science and riskbased lean strategy. However, nine companies reported success (i.e., approval of application) when pursuing such a strategy. Those who have reported success indicated that they reduced time points and/or quality attributes tested, and tested at long-term conditions only. To justify the proposed strategy, applicants used a mixture of statistical modeling, moisture vapor transfer rate (MVTR) data, and/or a stability risk assessment. Risk-based predictive stability has rarely been used to assess risk for a post-approval change.

Nine companies responded that they have received approval of reduced post-approval protocols in the US and/or globally for reductions in time points and quality attributes tested and in proposing long-term conditions only. However, industry respondents indicated significant divergence among global health authorities to the receptivity of lean stability and RBPS-based regulatory 


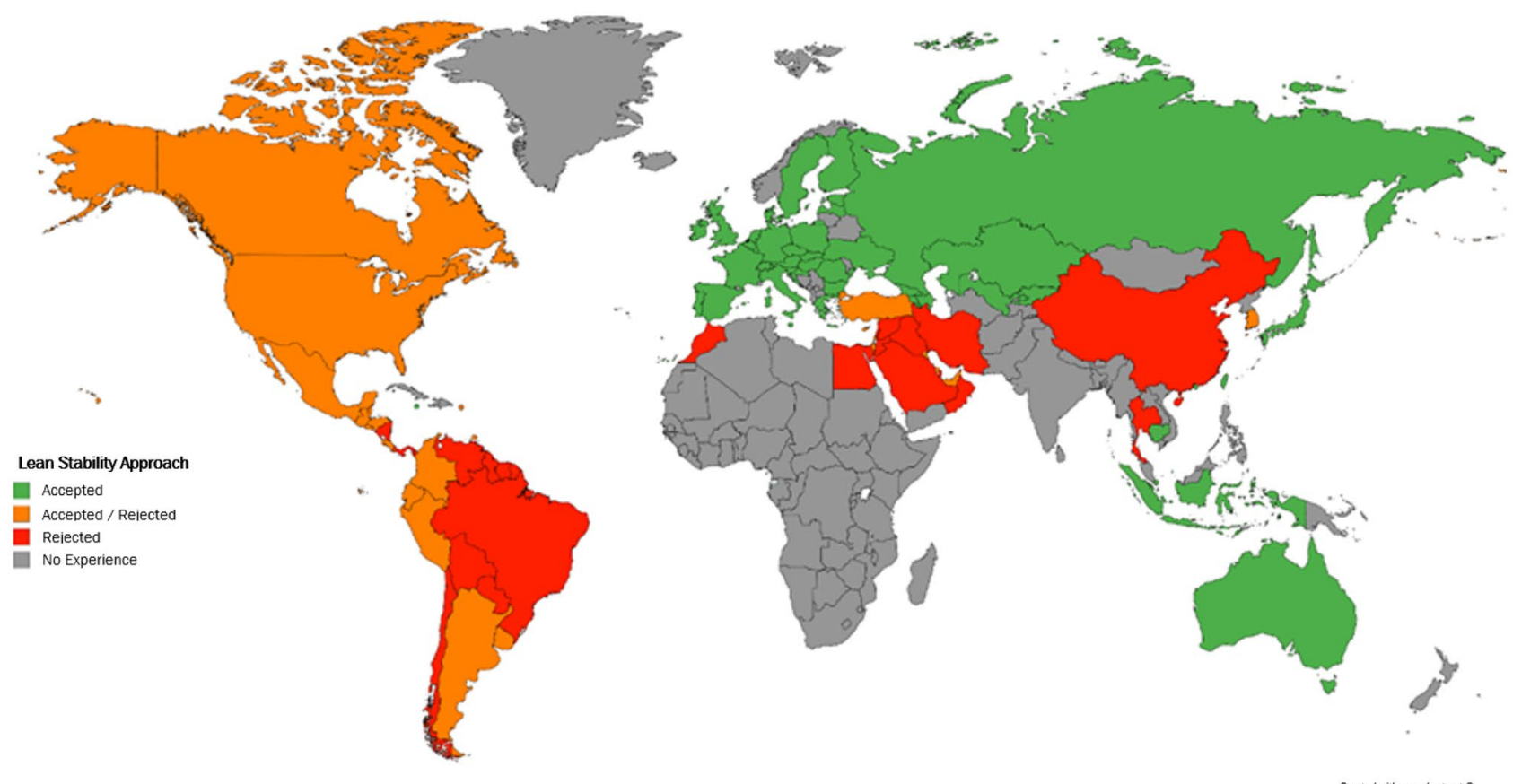

Fig. 3 Divergence among global health authorities to receptivity of lean stability approach(es) in registration and post-approval regulatory submission. Color legend: green countries' health authority(ies) were cited by survey respondent(s) as accepting lean stability strategies. Orange countries' health authority(ies) were cited by survey respondent(s) for accepting lean stability strategies in some cases and

submissions, as illustrated in Fig. 3. It is important to note that this illustration reflects the feedback of participants to the survey and is not intended to be predictive of the success of any future lean stability approaches filed to the various global authorities.

Examples of divergent thinking among global agencies, as indicated by the experiences shared by respondents, are summarized in Table 3. Survey responses also indicated inconsistency within the same health authority. rejecting them in other cases. Red countries' health authoritiy(ies) were cited by survey respondent(s) for rejecting lean stability strategies. Gray countries did not have any specific examples of health authority feedback to lean stability strategies cited by survey respondent(s)

Lack of clarity and divergence in expectations for stability data by global health authorities were cited as an obstacle to pursuing approval of lean stability approaches, and as illustrated in Table 3. Respondents were overwhelmingly supportive of a potential new ICH guideline under Q1 titled Science and Risk-based Approaches to Stability Testing for Post-Approval CMC Changes and Post-Approval Stability Commitments. Respondents also agreed it would be beneficial to industry to establish a harmonized guideline on

Table 3 Regulatory authorities reported by industry respondents to have not approved lean stability approach submissions for registration or post-approval commitments and changes

\begin{tabular}{|c|c|}
\hline Region & Agency reason(s) for not accepting lean stability approach(es) \\
\hline United States & $\begin{array}{l}\text { Insufficient historical stability data to justify the reduced testing frequency. Additional tests/timepoints required } \\
\text { (however, removal of some testing was accepted) }\end{array}$ \\
\hline Brazil & $\begin{array}{l}\text { Reduced models not allowed per country guidance. Matrix justification was questioned but not rejected at time of } \\
\text { survey }\end{array}$ \\
\hline China & Scrutiny of departure from ICH protocol and bracketing requirements \\
\hline South Korea & $\begin{array}{l}\text { Bracketing rejected for multi-strength drug product because the excipients did not scale proportionally to the } \\
\text { varying amounts of active ingredient }\end{array}$ \\
\hline Canada & $\begin{array}{l}\text { Objected to the exclusion of assay testing from stability monitoring. The reduced stability protocol was subse- } \\
\text { quently accepted after assay testing was added by the sponsor }\end{array}$ \\
\hline Latin America; Middle East & Expected full real-time data equivalent to the desired expiry period \\
\hline Thailand; Morocco; Bahrain & Additional/full data required; no specific scientific rationale stated by the health authority \\
\hline
\end{tabular}




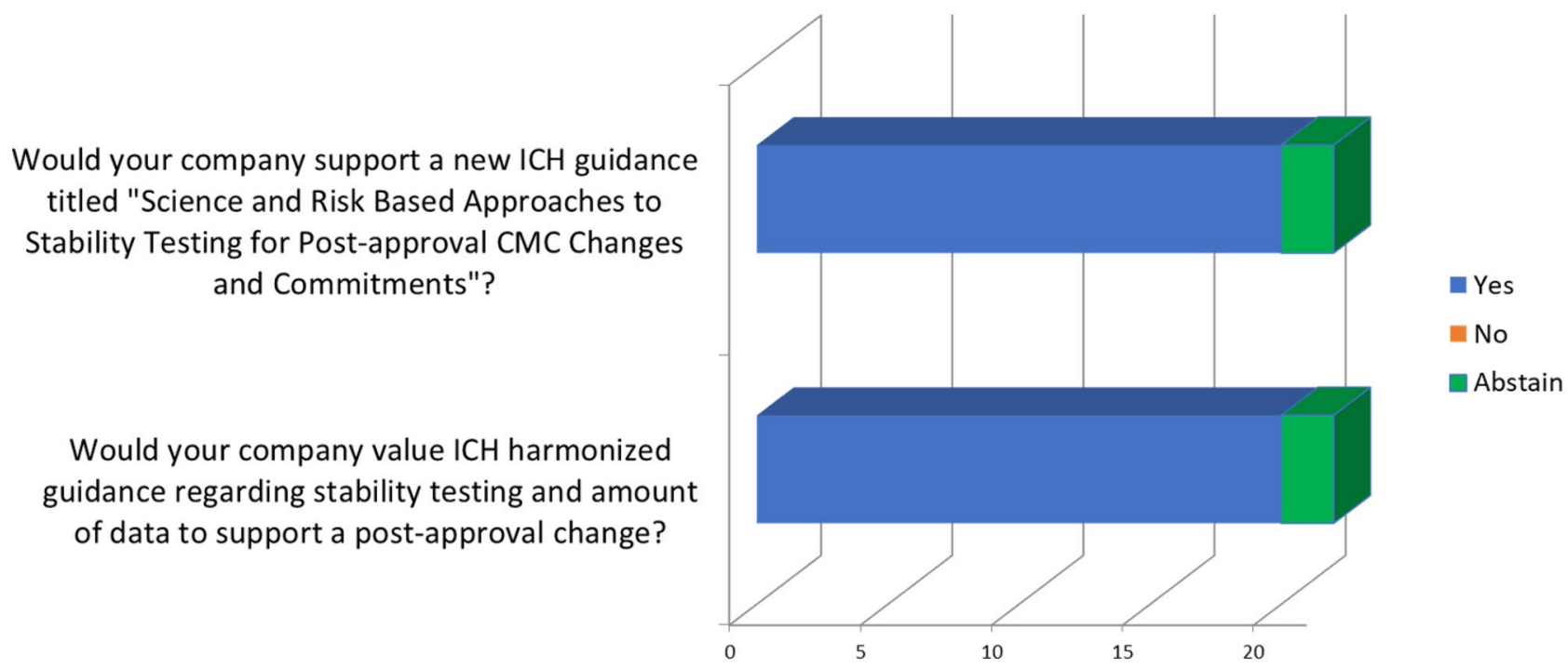

Fig. 4 Industry alignment regarding the development of new ICH guidance(s) to facilitate adoption of lean stability approach(es) agreeable to global regulatory agencies

stability testing and the amount of data required to support post-approval manufacturing changes under ICH. Refer to Fig. 4.

\section{Additional Topics}

\section{Bulk Drug Product Stability}

Responding companies who conduct bulk drug product stability studies during development overwhelmingly do so during the registration stage. Half of the respondents indicated that there are no procedural timelines nor project milestones associated with bulk stability studies, and that these studies are conducted across a variety of development stages, including clinical, registration, and post-approval/ life cycle.

Eight out of 18 respondents characterize bulk drug product stability in simulated packages, whereas 6 out of 16 respondents monitor in bulk drums. Four respondents use both packaging types. However, it is unclear if more than one type of bulk stability study is conducted for the same drug product, i.e. using both simulated packages and bulk drums.

\section{In-use Stability Studies}

In-use stability studies are conducted throughout the development phases for solid oral dosage forms, i.e. tablets or capsules, with little evidence of these studies being preferentially performed in any specific development phase.

However, the critical rationales associated with in-use stability studies for solid oral dosage forms are regulatory requirements (known or perceived, e.g., based on prior experience via health authority questions). Product risk is a consideration but is linked with the regulatory requirements. Of the 13 companies citing regulatory requirements as a reason for performing in-use stability studies, six cited known requirements, five cited known and perceived requirements, and two cited perceived requirements.

Similarly, for complex dosage forms, i.e., solution, suspension, reconstituted, or parenteral dosage forms, in-use stability studies are conducted throughout the development phases. For these dosage forms, however, there is greater variability in the stated rationales or drivers for conducting in-use stability studies. Regulatory requirements are the key drivers for in-use stability studies. However, other factors were also cited (refer to Table 4). This may be indicative of the dosage form complexity and subsequently a greater need to design individualised in-use stability studies.

\section{Placebo to Match}

Regarding type of placebo to match (PTM) drug product used to support clinical trials, most companies use an analytical PTM, i.e., one that is identical to the excipient composition of the active drug product on a quantitative basis (10 out of 23 responses). Five companies use a generic PTM approach, i.e., one that is identical to the excipient composition of the active drug product on a qualitative basis or that is identical visually but varies in excipient composition (uses different excipients) relative to the active drug product. Eight companies indicate that they use both analytical and generic PTM dosage forms. 
Table 4 Rationales for conducting in-use stability studies on complex dosage forms

\begin{tabular}{ll}
\hline Rationale & $\begin{array}{l}\text { Number of } \\
\text { responses }\end{array}$ \\
\hline Regulatory requirement & 8 \\
Product risk (knowledge based on SRA) & 5 \\
Clinical use/patient use & 4 \\
Perceived regulatory requirement & 2 \\
Prior queries & 2 \\
Label claim/patient leaflet & 2 \\
Product knowledge & 2 \\
Internal QA expectation & 1 \\
Internal procedure & 1 \\
Business risk mitigation & 1 \\
\hline
\end{tabular}

A high number of responses indicated that these PTM dosage forms are placed on stability to support shelf life dating (16 out of 23 responses). The majority of PTM dosage form stability studies are designed to match those for the corresponding active drug product with other approaches employed. Since most survey respondents indicated their focus as small molecule pharmaceutical products and there were only four responses specifically designated for biological/ biotechnological products, it was not possible to differentiate PTM practices between these modalities (Table 5).

Responses were varied regarding attributes typically assessed during testing and ranged from appearance/ description testing only being performed to full physical and microbiological analyses.

Six companies indicated that they do not conduct PTM stability studies at all. To justify PTM shelf-life dating, these companies use the stability data generated on the active drug product (4 responses), use universal (generic-to-match) placebo data (1 response), or use a default 5 -year shelf life dating (1 response).

\section{Discussion}

\section{Industry Divergence}

The survey responses demonstrated the progress made since the introduction of ICH Guidelines Q8-11 and their accompanying science and risk-based frameworks for the drug development lifecycle. The rise in use of predictive stability modelling tools in early development is indicative of this change. While encouraging progress is evident, the survey also highlighted divergences in the stability strategies employed by participating companies that suggest a continuing reliance on legacy empirical stability models.

For example, the prevalence of stability risk assessment usage is a strong finding with over $70 \%$ of companies either using or intending to use this approach. However, stability risk assessments vary considerably in format and focus, from general assessments of existing data to formalized risk scoring. Similarly, the tests performed during stability studies not only include the expected stability-related and shelf-life limiting attributes but also a range of additional tests. These tests are often risk management measures, built into protocols to mitigate against anticipated regulatory questions.

Another example of the variability in adoption of lean stability strategies in the participating companies can be found in approaches toward defining shelf-lives for placebo products. The survey shows that the predominant approach is to conduct stability studies on analytical placebos to match, with test protocols as extensive as those performed on the active drug product. Yet, some participating companies have successfully used a default shelf-life dating approach.

There are various reasons behind the differences in practices between companies, but one theme emerges from the survey feedback: concern over regulatory risk, perceived or otherwise. This concern may be grounded in previous experience, a reluctance to engage with agencies in
Table 5 Approaches to PTM stability study design

\begin{tabular}{ll}
\hline Study design for PTM & $\begin{array}{l}\text { Number of } \\
\text { responses }\end{array}$ \\
\hline Match design to active drug product & 8 \\
Study design as per ICH Q1A & 4 \\
Long-term storage condition only with annual testing to shelf-life & 2 \\
Generic PTM only placed on stability & 1 \\
Design based on formulation knowledge and limited timepoints/storage conditions/testing & 1 \\
used & \\
\hline
\end{tabular}


science-based discussions, or mitigation against anticipated regulatory queries. There may also be differences in receptiveness towards risk within companies leading to conservative strategies where some stakeholders strive to minimize regulatory queries.

Nonetheless, the impact of industry divergences in stability strategy manifests itself in increased costs to pharmaceutical companies in terms of materials, facilities, time and workforce demands. In addition, traditional ICH Q1A and Q5C stability approaches require the use of highly skilled workforces on routine analyses that may be unnecessary, instead of focusing efforts onto strategies that balance science and compliance.

The way forward in addressing the internal variabilities lies in enhanced knowledge-sharing across industry. Several case studies documenting successful adoption of lean stability approaches are available in the external literature [8-16]. These examples can drive change within industry as the strategies described become embedded in quality documentation and processes. Consequently, consistent application of science- and risk-based lean stability approaches in regulatory submissions will aid acceptance by external health authorities and agencies.

\section{Health Authority Divergence}

Advances in science and industry practices, coupled with development of modeling tools, have resulted in improved understanding of attributes that contribute to drug stability. These advancements have enabled stability predictions and development of optimized protocols including test attributes with a direct impact only on the stability of the drug. Additionally, these tools provide the ability to assign and extend shelf-life without realtime data availability. Regulatory agencies benefit from adoption of lean stability strategies because the relevant Module 3 sections focus only on stability-indicating quality attributes. This, in turn, leads to more efficient review processes within agencies ultimately enabling continual lifecycle improvement within the industry. Lean stability strategies also require increased understanding of the drug substances and drug products in question. This will benefit both industry and regulatory agencies by mitigating the risk of encountering unexpected findings during development and post-approval.

Survey results indicate that the divergences are not confined to the implementation of these strategies within the industry but are also present in how they are accepted by global regulatory agencies. Examples of divergence amongst regulatory agencies are noted in Table 3. This lack of acceptance might be attributed to multiple factors: lack of regulatory guidance, local regulations limiting the acceptability of lean approaches, and the need for additional details in the application describing the tools used to justify lean approaches, to name a few. If even one health authority objects to a proposed lean stability approach, a company may feel obliged to apply the most conservative stability protocol globally.

Current guidance documents and regulatory guidelines acknowledge alternative approaches, but do not elaborate on risk-based and lean strategies to stability. Increasing understanding between industry and regulatory authorities is critical in advancing the science and achieving acceptance of these approaches. The value of harmonized guidance around risk-based lean stability approaches is strongly supported by the industry. By having globally acceptable approaches to stability using lean strategies, the time to develop and deliver drugs to the patient without compromising drug safety/efficacy can be minimized.

\section{Opportunities for Increased Industry-Agency Awareness}

Lean stability strategies can be applied during all stages of drug development. However, the regulatory acceptance of these strategies is variable. Despite the challenges of wider implementation and acceptability of lean stability strategies, some of the following opportunities exist for industryregulatory agency interaction.

Key feedback from interaction of IQ Consortium working group members with the MHRA in October 2018 and with ANVISA and other Latin American health authorities in March 2019 indicate that agencies are interested in industry presenting science and risk-based stability case studies to them. This is most impactful for those health authorities relatively new to ICH who wish to grow their technical capability in stability science. Increased inclusion of predictive and lean strategies within regulatory filings will increase agency familiarity and knowledge of these approaches. Also, case study sharing across pharma companies will enable more informed decisions on use of risk-based stability strategies during development and post-approval phases of a product's life cycle and greater consistency in stability strategies presented in regulatory filings. Industry also has a critical role to play in continued communication through consortia influential in development and revision of ICH guidelines.

In addition, it is advantageous for health authorities to send representation to stability topic conferences to ensure awareness of industry trends and to enable dialogue between regulators and industry. Conferences also provide a collaborative environment for cross-industry workshops at which successes and learnings can be shared for the benefit of all. 


\section{Conclusion}

While global implementation and acceptance of lean stability strategies is variable, the industry and regulators have recognized the need for stronger understanding and are moving in the right direction. Pharmaceutical companies should continue prioritizing opportunities to engage global health authorities with lean stability case studies through regulatory submissions, agency engagements, and conferences. Stability guidance also needs to be updated to include risk-based principles that are scientifically sound and ensure safety and efficacy of the product through shelflife. Reducing the burden for stability studies, without compromising the quality of data and understanding of the product, can accelerate delivery of innovative medicines to the clinic and to the market and enable continual life cycle improvements. The advances in lean stability strategies can be realized through education and collaboration between industry and global regulatory agencies. Implementation of these strategies will benefit the industry, regulatory agencies, and most importantly patients.

Funding This paper was developed with the support of the International Consortium for Innovation and Quality in Pharmaceutical Development (IQ, www.iqconsortium.org). IQ is a not-for-profit organization of pharmaceutical and biotechnology companies with a mission of advancing science and technology to augment the capability of member companies to develop transformational solutions that benefit patients, regulators and the broader research and development community.

Data Availability All case study information provided have been approved by the contributing company/author.

\section{Declarations}

Conflict of Interest The authors declare that they have no conflict of interest.

Open Access This article is licensed under a Creative Commons Attribution 4.0 International License, which permits use, sharing, adaptation, distribution and reproduction in any medium or format, as long as you give appropriate credit to the original author(s) and the source, provide a link to the Creative Commons licence, and indicate if changes were made. The images or other third party material in this article are included in the article's Creative Commons licence, unless indicated otherwise in a credit line to the material. If material is not included in the article's Creative Commons licence and your intended use is not permitted by statutory regulation or exceeds the permitted use, you will need to obtain permission directly from the copyright holder. To view a copy of this licence, visit http://creativecommons.org/licenses/by/4.0/.

\section{References}

1. International Conference for Harmonisation, ICH Press Release. "Continued advancement in global harmonisation efforts as ICH prepares for 30 year commemoration.” 27 November 2019. https:// admin.ich.org/sites/default/files/inline-files/ICH39Singapore_ PressRelease_2019_1127_Final_4.pdf, Accessed 23 Mar 2020.

2. ICH: Guideline Q1A(R2) Stability Testing of New Drug Substances and Products. Step 4 version. 2003 https://database.ich. org/sites/default/files/Q1A\%28R2\%29\%20Guideline.pdf.

3. ICH: Guideline Q5C Quality of Biotechnological products: Stability testing of biotechnological/biological products. Step 4 version. 1995 https://database.ich.org/sites/default/files/Q5C\% 20Guideline.pdf.

4. ICH: Guideline Q1D Bracketing and matrixing designs for stability testing of new drug substances and products. Step 4 version. 2002 https://database.ich.org/sites/default/files/Q1D\% 20Guideline.pdf.

5. ICH: Guideline Q1E Evaluation for stability data. Step 4 version. 2003 https://database.ich.org/sites/default/files/Q1E\%20Guideline.pdf.

6. ICH: Guideline Q6A Specifications: Test procedures and acceptance criteria for new drug substances and new drug products: Chemical substances. Step 4 version. 1999 https://database.ich. org/sites/default/files/Q6A\%20Guideline.pdf.

7. ICH: Guideline Q6B Specifications: test procedures and acceptance criteria for biotechnological/biological products. Step 4 version.' 1999 https://database.ich.org/sites/default/files/Q6B\% 20Guideline.pdf.

8. Freed AL, Clement E, Timpano R. Regulatory responses to the use of various lean stability strategies in early drug development. Regul Rapporteur. 2014;11:5-8.

9. Freed, AL. Lean stability: Global regulatory reception - successes and challenges of recent case studies. AAPS (Washington): 05-April 2016.

10. Colgan S, Hofer J, Timpano R, Vukovinsky K, Waterman K, Norris K. Lean stability. AAPS Newsmagazine Sept. 2015, 14-16.

11. Colgan ST, Timpano RJ, Roberts M, Weaver R, Ryan K, Fields K, Scrivens G. Opportunities for lean stability strategies. J Pharm Innov. 2014a. https://doi.org/10.1007/s12247-014-9198-x.

12. Waterman KC, Carella AJ, Gumkowski MJ, Lukulay P, MacDonald BC, Roy MC, Shamblin SL. Improved protocol and data analysis for accelerated shelf-life estimation of solid dosage forms. Pharm Rsrch. 2007;24:780-90.

13. Clancy D, Hodnett N, Orr R, Owen M, Peterson J. Kinetic model development for accelerated stability studies. AAPS PharmSciTech. 2017;18:1158-76.

14. Qiu F, Scrivens G, editors. Accelerated predictive stability (APS): fundamentals and pharmaceutical industry practices. 1st ed. Cambridge, MA, USAA: Academic Press; 2018.

15. Colgan ST, Watson TJ, Whipple RD, Nosal R, Beaman JV, Antonis DMD. The application of science-and risk-based concepts to drug substance stability strategies. J Pharm Innov. 2012;7(3-4):205-13.

16. Colgan ST, Timpano RJ, Roberts M, Weaver R, Ryan K, Fields $\mathrm{KW}$, Scrivens G. Opportunities for lean stability strategies. J Pharm Innov. 2014b;9(4):259-71.

17. Hyzer CH, Adamec E, Catacchio B, Coon A, Dill A, Fu M, Gruenig L, Lesslie M, McMahon M, Pulliam D, Qiu F, Regler BP, Szymulanska-Ramamurthy K, Timpano R, Wannere C. Lean stability case studies-leveraging science- and risk-based approaches to enable meaningful phase specific pharmaceutical stability strategies. J Pharm Innov. 2020. https://doi.org/10.1007/ s12247-020-09463-z.

Publisher's Note Springer Nature remains neutral with regard to jurisdictional claims in published maps and institutional affiliations. 